\title{
Dynamic interplay between locus-specific DNA methylation and hydroxymethylation regulates distinct biological pathways in prostate carcinogenesis
}

Shivani N. Kamdar ${ }^{1,2}$, Linh T. Ho ${ }^{1}$, Ken J. Kron ${ }^{1}$, Ruth Isserlin³ ${ }^{3}$ Theodorus van der Kwast ${ }^{1,4}$, Alexandre R. Zlotta ${ }^{5}$, Neil E. Fleshner ${ }^{6}$, Gary Bader ${ }^{3}$ and Bharati Bapat ${ }^{1,2^{*}}$ (D)

\begin{abstract}
Background: Despite the significant global loss of DNA hydroxymethylation marks in prostate cancer tissues, the locus-specific role of hydroxymethylation in prostate tumorigenesis is unknown. We characterized hydroxymethylation and methylation marks by performing whole-genome next-generation sequencing in representative normal and prostate cancer-derived cell lines in order to determine functional pathways and key genes regulated by these epigenomic modifications in cancer.

Results: Our cell line model shows disruption of hydroxymethylation distribution in cancer, with global loss and highly specific gain in promoter and CpG island regions. Significantly, we observed locus-specific retention of hydroxymethylation marks in specific intronic and intergenic regions which may play a novel role in the regulation of gene expression in critical functional pathways, such as BARD1 signaling and steroid hormone receptor signaling in cancer. We confirm a modest correlation of hydroxymethylation with expression in intragenic regions in prostate cancer, while identifying an original role for intergenic hydroxymethylation in differentially expressed regulatory pathways in cancer. We also demonstrate a successful strategy for the identification and validation of key candidate genes from differentially regulated biological pathways in prostate cancer.

Conclusions: Our results indicate a distinct function for aberrant hydroxymethylation within each genomic feature in cancer, suggesting a specific and complex role for the deregulation of hydroxymethylation in tumorigenesis, similar to methylation. Subsequently, our characterization of key cellular pathways exhibiting dynamic enrichment patterns for methylation and hydroxymethylation marks may allow us to identify differentially epigenetically modified target genes implicated in prostate cancer tumorigenesis.
\end{abstract}

Keywords: 5-Hydroxymethylcytosine, 5-Methylcytosine, Whole-genome, Prostate cancer, Cell lines, Integrative analysis

\footnotetext{
* Correspondence: bapat@lunenfeld.ca

'Department of Laboratory Medicine and Pathobiology, University of

Toronto, Toronto, ON, Canada

${ }^{2}$ Mount Sinai Hospital, Lunenfeld-Tanenbaum Research Institute, Toronto,

ON, Canada

Full list of author information is available at the end of the article
} 


\section{Background}

Tumorigenesis is regulated by a cascade of genetic and epigenetic alterations, with aberrant cytosine base methylation $(5 \mathrm{mC})$ acting as one of the key defining characteristics of tumor cells. CpG islands (CGIs), characterized by clusters composed of cytosine nucleotides followed by guanines, are usually unmethylated in normal conditions. However, in cancer, locus-specific hypermethylation of CGIs, particularly in the promoter regions of tumor suppressor genes, results in their loss of expression [1-3]. Simultaneously, genome-wide global hypomethylation of repetitive sequences in tumors results in genomic instability, promoting chromosomal rearrangement and the reactivation of transposable elements $[4,5]$.

5-Hydroxymethylated marks $(5 \mathrm{hmC})$ were first characterized in mammalian genomes as transient intermediates in the process of DNA demethylation [6]. However, the recent discovery of $5 \mathrm{hmC}$ as a stable epigenetic mark that also shows global loss in solid tumors and hematological malignancies $[7,8]$ has opened up new avenues for investigation into the dynamics of epigenetic regulation in cancer. $5 \mathrm{hmC}$ shows striking differences in distribution patterns among human tissues, exhibiting very high content in the brain and low content in the blood, spleen, and placental tissue [9, 10]. Genomic $5 \mathrm{hmC}$ distribution also differs by region, showing enrichment at exon-intron boundaries, exons, promoters, and enhancer elements [11-14]. Generally, the presence of $5 \mathrm{hmC}$ marks is associated with increased expression $[14,15]$; however, the role of promoter hydroxymethylation in regulating expression may differ based on cell type [16].

Several pieces of evidence suggest a key role for $5 \mathrm{hmC}$ in governing tumorigenesis. Firstly, the genome-wide loss of $5 \mathrm{hmC}$ in cancer cannot be completely explained by the corresponding global loss of $5 \mathrm{mC}$, indicating an independent role for $5 \mathrm{hmC}$ alterations in tumors [7]. Secondly, $5 \mathrm{hmC}$ correlates directly with differentiation state in cells during development, and its loss may thus dispose tumor cells toward uncontrolled proliferation $[17,18]$. Furthermore, TET enzymes, which oxidize $5 \mathrm{mC}$ to produce $5 \mathrm{hmC}$, often exhibit mutations or transcriptional downregulation in many different types of cancers, especially in hematological malignancies and gliomas [18-21]. The dioxygenase activity of TET proteins is dependent on the presence of $\alpha$-ketoglutarate, which acts as a catalytic cosubstrate for $5 \mathrm{hmC}$ production [22]. Intriguingly, isocitrate dehydrogenase enzymes 1 (IDH1) and 2 (IDH2), which are normally able to produce $\alpha$ ketoglutarate through the decarboxylation of isocitrate, are mutated in many human cancers. $I D H$ mutations not only inhibit their ability to produce $\alpha$-ketoglutarate but result in the production of the oncometabolite 2- hydroxyglutarate (2HG), which is able to directly inhibit the activity of TET proteins [20,22, 23].

Thus, $5 \mathrm{hmC}$ patterning across the tumor genome may act as a hallmark of cancer development and progression. However, the locus- and gene-specific roles of $5 \mathrm{hmC}$ and their significance in tumorigenesis have not yet been well characterized in non-neuronal solid tumors, including prostate cancer (PCa).

$\mathrm{PCa}$ is the most common malignancy and the second highest cause of death from cancer in men worldwide [24]. Currently, the gold standard for PCa diagnosis is prostate-specific antigen (PSA) testing. However, due to its high false positive detection rate and the inability of PSA levels to differentiate between indolent and aggressive disease, the widespread usage of PSA screening has resulted in frequent overdiagnosis and overtreatment of the disease, an issue made critical by the significant morbidity associated with radical treatment [25-27]. This issue is further complicated by extensive tumor multifocality and heterogeneity. A majority of $\mathrm{PCa}$ patients present with multiple nonclonal foci of disease, several of which may possess differential histologic grades. Thus, genetic heterogeneity in $\mathrm{PCa}$ is not only widespread between patients but also within single prostate tumor specimens. Individual patients may thus possess multiple distinct genomic profiles at each tumor focus, complicating $\mathrm{PCa}$ diagnosis, prognostication, and development of treatment strategies [28-30].

Epigenetic alterations also contribute to $\mathrm{PCa}$ tumor heterogeneity, with locus-specific variability in cytosine base methylation $(5 \mathrm{mC})$ occurring concurrently with copy-number alterations in primary prostate tumors [31]. Although global hypomethylation changes are a late event in $\mathrm{PCa}$, associated with metastatic progression [32], promoter hypermethylation is an early and frequent event in prostate carcinogenesis. Hypermethylationinduced silencing of the tumor suppressor glutathione S-transferase P1 gene (GSTP1) is the most frequent DNA aberration in $\mathrm{PCa}$, while promoter hypermethylation of GSTP1, RASSF1A (Ras association domain family 1 isoform $\mathrm{A}$ ), and $A P C$ (adenomatous polyposis coli) correlates with PCa stage [33, 34].

Thus, comprehensive characterization of the $5 \mathrm{hmC}$ modifications underlying $\mathrm{PCa}$ development and, consequently, the mechanisms of epigenetic regulation in $\mathrm{PCa}$ may help to address these issues. Investigation of differential patterns of $5 \mathrm{hmC}$ and $5 \mathrm{mC}$ distribution across genomic features (intergenic, intronic, exonic, promoter, DNAse I hypersensitive site, or CGI regions) could provide important insights into prostate cancer pathogenesis.

In this study, we aim to determine the biological role of aberrant $5 \mathrm{hmC}$ patterning in regulating biological pathways and key candidate genes in prostate 
tumorigenesis. We have used a whole-genome nextgeneration sequencing strategy to investigate the dynamic interplay between locus-specific $5 \mathrm{mC}$ and $5 \mathrm{hmC}$ marks and their relationship with altered gene expression, performing one of the first integrative analyses of its kind in prostate cancer. Our data shows that $5 \mathrm{hmC}$ marks display differential effects on gene regulation based on locus-specific changes unique to cancer cells and that it may play a critical role in governing central tumorigenic pathways related to signaling and cellular proliferation in prostate cancer.

\section{Results}

Genome-wide locus-specific hypermethylation and global reduction of hydroxymethylation occurs in prostate cancer cells compared to normal prostate

In order to investigate the distribution of $5 \mathrm{mC}$ and $5 \mathrm{hmC}$ in the prostate cancer genome, we performed next-generation sequencing (NGS) following methyl-binding protein capture (MBD-seq, $n=3$ ) and hydroxymethyl-selective chemical labeling (hMeSeal-seq, $n=1$ ) in a representative normal prostate tissue-derived cell line (RWPE-1) and a xenograftderived prostate carcinoma cell line (22Rv1). Profiling of these epigenetic marks across RefSeq-annotated genes revealed dramatic differential hypermethylation across all genomic features in 22Rv1 as compared to RWPE-1, with gene expression lowering in concordance with increased methylation peak density. In contrast, marked global loss of absolute $5 \mathrm{hmC}$ was noted across the 22Rv1 genome (Additional file 1: Figure S1), while locus-specific alterations of both marks could be observed across genes (Fig. 1).
$5 \mathrm{mC}$ and $5 \mathrm{hmC}$ show differential locus-specific abundance in prostate cancer

As locus-specific $5 \mathrm{hmC}$ is poorly characterized in prostate cancer, and due to the lowered distribution of $5 \mathrm{hmC}$ in cell culture [35], we first set out to determine the validity of our cell line hydroxymethylation profiling. hMeSeal-seq samples chosen for analysis displayed significant normalized read enrichment compared to input samples (Additional file 1: Figure S2), while dot blot analysis of genomic DNA from both cell lines showed corresponding evidence of $5 \mathrm{hmC}$ (Additional file 1: Figure S3). Furthermore, the proportion of promoter, intergenic, and CGI hydroxymethylation in both cell lines is significantly lower than one would expect from random genomic distribution of the $5 \mathrm{hmC}$ mark across CpGs, indicating the presence of locus-specific patterning in our samples (Additional file 1: Table S1). Validation of hMeSeal kit specificity was also performed via the testing of control oligomers (Additional file 1: Figure S4). Finally, in order to validate our $5 \mathrm{hmC}$ marks in RWPE-1, we performed hydroxymethylated DNA immunoprecipitation sequencing (hMeDIP-seq) on a separate biological replicate $(n=1)$ from the same normal cell line. Specificity of the hMeDIP antibody was verified via internal spike-in of control sequences (Additional file 1: Table S2). We found that $55.8 \%$ of hydroxymethylated gene regions (6324 unique genes) detected by our hMeSeal technique were also detected in hMeDIP-seq, of which $4.1 \%$ (representing 257 genes) bore $5 \mathrm{hmC}$ peaks with at least partial overlap in exactly the same $0.48-1.5-\mathrm{kbp}$ regions (Fig. 2a). hMeDIP-seq samples were sonicated separately from

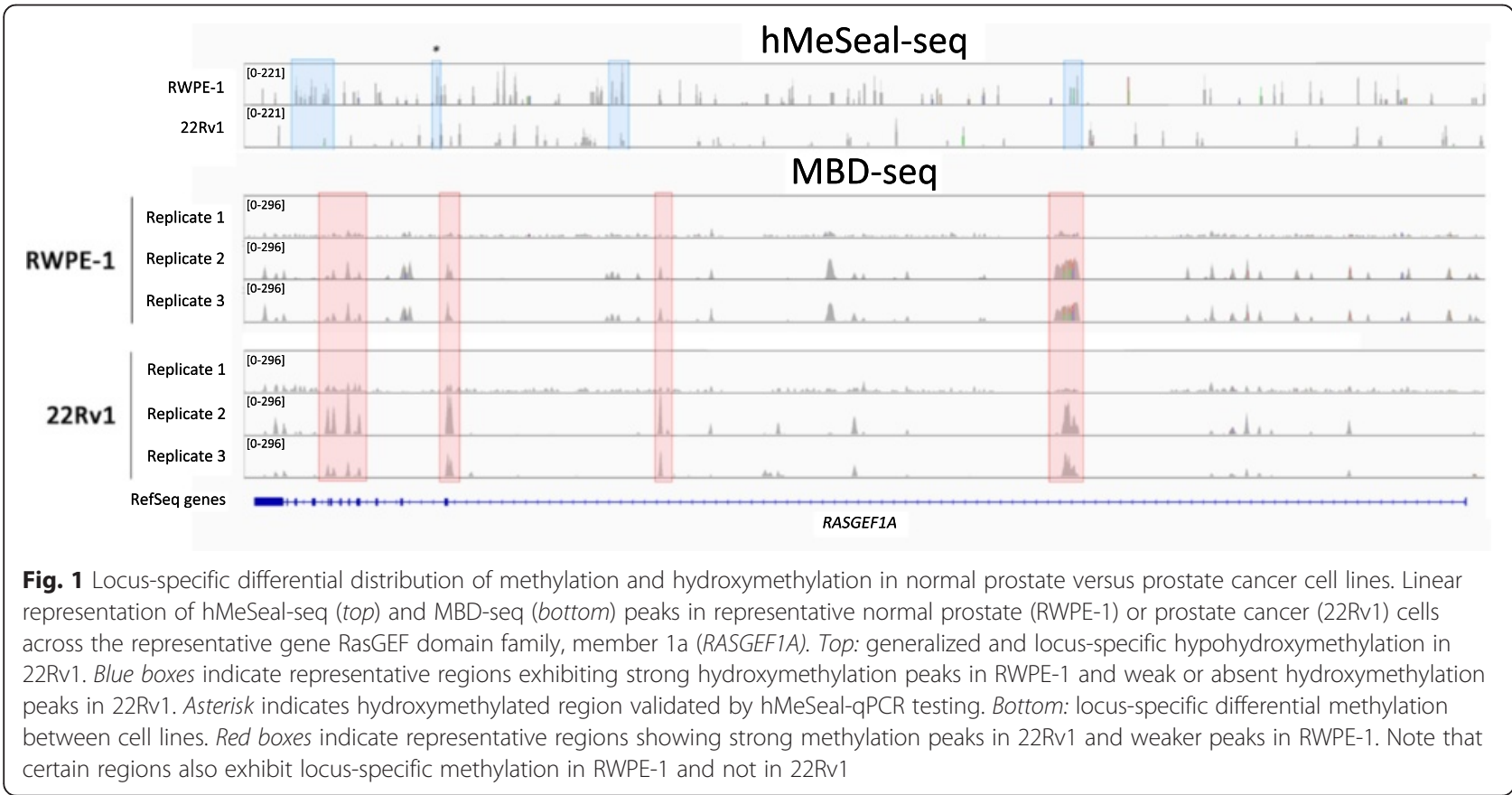




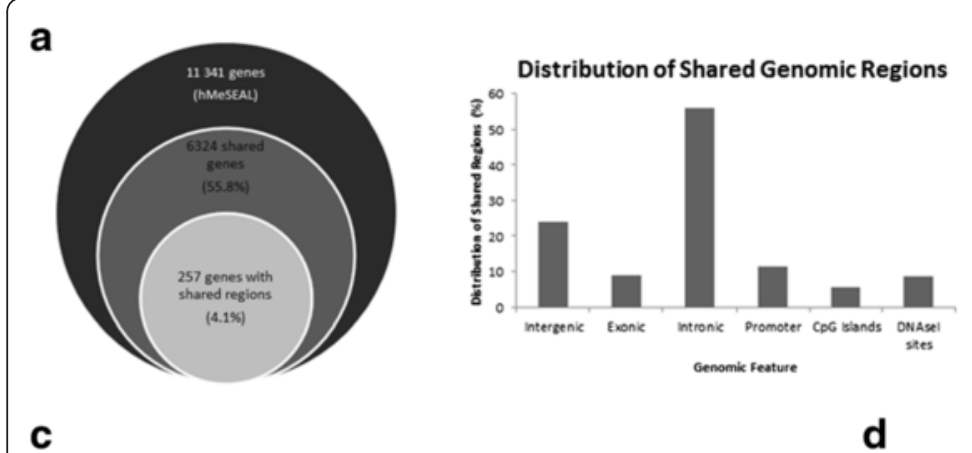

b
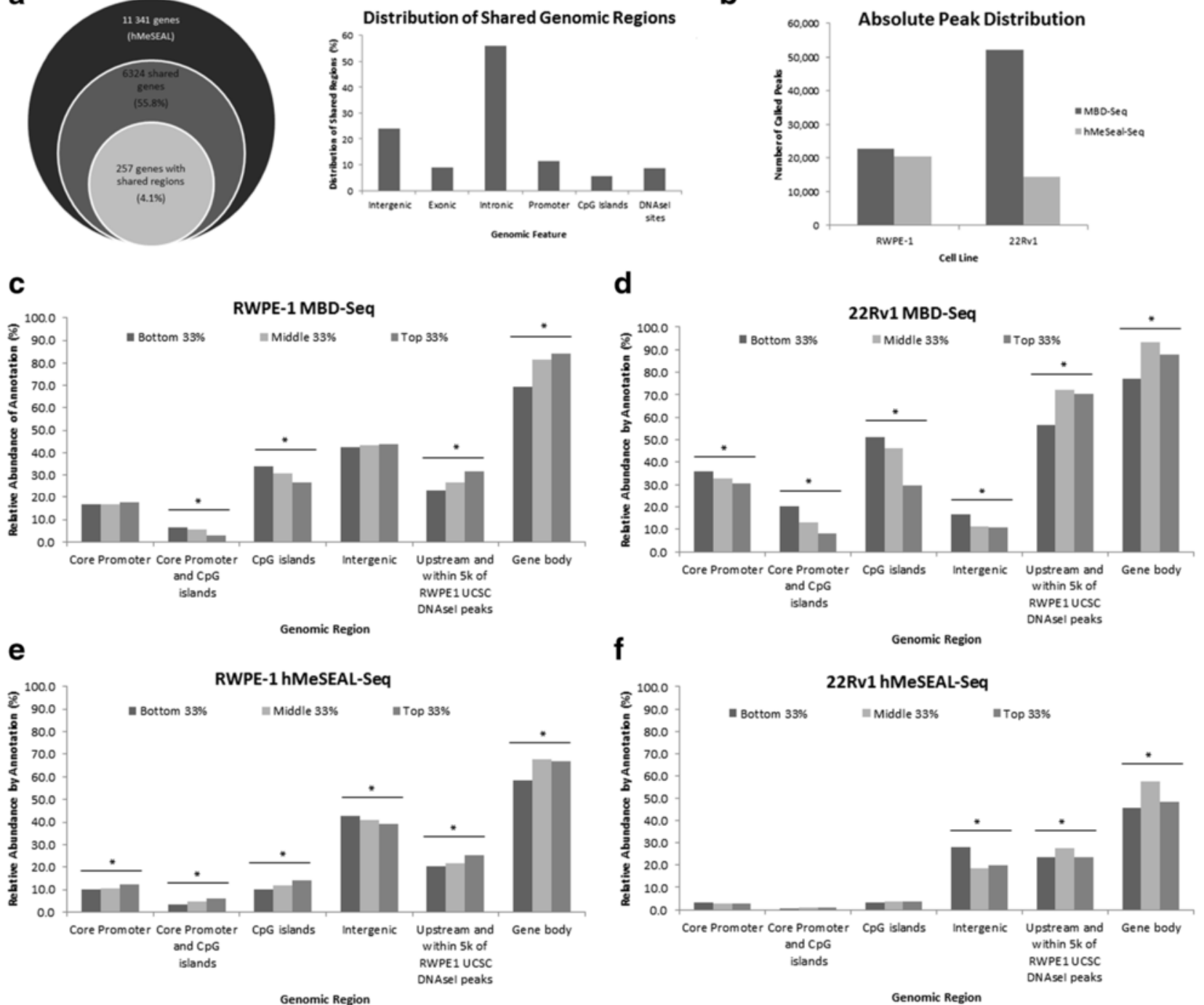

Fig. 2 Correlation between locus-specific methylation or hydroxymethylation and gene expression. a Validation of hMeSeal-Seq results by hMeDIPSeq. Left: $62.6 \%$ of genes detected as hydroxymethylated in RWPE-1 by hMeSeal were also detected as hydroxymethylated using hMeDIP, while $2.3 \%$ of peaks from these genes were called in exactly the same chromosomal location using both methods. Right: genomic feature distribution for peaks called in the same regions in both hMeDIP- and hMeSeal-seq. Some features may overlap each other, and thus one peak may be accounted for more than once. b Total peaks called from sequencing data in normal and prostate cancer cell lines. Peaks called across three replicates for MBD-Seq or within one single replicate for hMeSeal-seq. $\mathbf{c}-\mathbf{f}$ Differentially methylated $(\mathbf{c}, \mathbf{d})$ and differentially hydroxymethylated $(\mathbf{e}, \mathbf{f})$ regions for both cell lines stratified by genomic feature. Bar graphs depict the relative abundance of each mark within each of three expression tiers from microarray analysis. Asterisks represent significant $p$ values ( $p<0.05$, chi-square test for trend) indicating correlation between the presence of $5 \mathrm{mC}$ or $5 \mathrm{hmC}$ marks in a given region and gene expression within each cell line. Upward trend of bars, when significant, indicates a positive correlation of a locus-specific mark with gene expression. Significant downward trend indicates negative correlation

hMeSeal-Seq samples, resulting in a different pattern of random shearing and making it highly unlikely that the observed overlap was generated by random chance. Peak distribution in both techniques did not significantly differ in all genomic features with the exception of DNAse I hypersensitive sites (DHSs), further confirming our hMeSeal-seq results (Additional file 1: Figure S5).

Next, we compared the abundance of differential methylation and hydroxymethylation peaks proximal to RefSeq genes between both cell lines (Fig. $2 \mathrm{~b}$ ), validating the specificity of MBD capture through quantitative PCR (qPCR) (Additional file 1: Figure S6). 22Rv1 had more than twice the number of differentially methylated annotated peaks or differentially methylated regions (DMRs) compared to RWPE-1. However, the locus-specific distribution of methylation marks differed between cell lines, with significant increases in the proportion of $5 \mathrm{mC}$ marks in CGIs and DHSs in cancer (Additional file 1: Figure S7A). DHSs 
are indicative of open chromatin and gene regulatory elements [36], and we obtained this dataset for RWPE-1 only. We observed significantly less proportional promoter methylation in 22Rv1 as compared to RWPE-1. These findings contrast with typical patterns of promoter hypermethylation in cancer and may potentially indicate site-specific derepression of certain genes. In contrast, the proportion of promoter CGI methylation was significantly greater in 22Rv1 than in RWPE-1, in accordance with the conventional methylation paradigm in cancer.

22Rv1 differential hydroxymethylation was significantly reduced in both overall peak number and proportionality of enrichment in exonic, promoter, CGI, and RWPE-1 DHS genomic features. However, proportional levels of intronic hydroxymethylation did not significantly differ between cell lines. Intriguingly, proportional intergenic hydroxymethylation was significantly greater in cancer compared to normal cells (Additional file 1: Figure S7B). Furthermore, pathway-based analysis of intergenic hydroxymethylation revealed extensive differential functional enrichment between normal and cancer cell lines, suggesting a putative role for intergenic $5 \mathrm{hmC}$ in the aberrant upregulation of proliferation androgen receptor signaling in cancer. While most literature to date has focused on pathological $5 \mathrm{hmC}$ loss, our results suggest a putative functional role for the presence or gain of hydroxymethylation marks in cancer.

\section{A novel negative correlation of $5 \mathrm{hmC}$ with expression in gene-proximal intergenic regions}

In order to determine the effect of the observed differential epigenetic mark distribution in 22Rv1 on gene regulation, we performed integrative analysis correlating (hydroxy)methylation-enriched genes stratified by genomic feature to gene expression levels obtained from publicly available microarray data for RWPE-1 [Gene Expression Omnibus (GEO) Accession: GSM375783] $(n=1)$ and 22Rv1 [GEO Accession: GSE36135] $(n=3)$. We divided the expression data into three equal tiers representing low, median, and high relative expression and correlated genes significantly differentially enriched for either $5 \mathrm{mC}$ or $5 \mathrm{hmC}$ marks to expression within each tier (Additional file 1: Table S3 and S4).

We found gene expression to exhibit negative correlation with methylation enrichment in CGIs (both within and outside core promoter regions) and positive correlation with methylation in genic regions and regions within $5 \mathrm{kbp}$ of UCSC RWPE-1 DHSs in both cell lines, as expected (Fig. 2c, d). Correlations between $5 \mathrm{mC}$ and expression tended to be more robust in the cancer cell line, with strong negative correlation to expression also observed for intergenic and promoter methylation alone in $22 \mathrm{Rv} 1$.
Significantly, we observed several novel correlations between hydroxymethylation and gene expression in the prostate. Hydroxymethylation enrichment in promoters, CGIs, and CGIs within the core promoter region correlated positively with gene expression in normal prostate, while a similar correlation with expression in these features was not observed in cancer (Fig. 2e-f). These findings contrast with the proportional gain of promoter and CGI $5 \mathrm{hmC}$ observed in pancreatic cancer [37]. Intriguingly, however, we observed a novel negative correlation between hydroxymethylation enrichment in intergenic regions and expression of the genes with transcription start sites (TSS) closest to the $5 \mathrm{hmC}$ mark. This correlation was strengthened in cancer, in contrast to the weakening of all other $5 \mathrm{hmC}$ expression trends. Furthermore, while $5 \mathrm{hmC}$ in genic regions and within $5 \mathrm{kbp}$ of UCSC RWPE-1 DHSs exhibited linear positive correlation with gene expression in normal prostate (similar to $5 \mathrm{mC}$ ), this correlation was strongly altered in prostate cancer, where the presence of hydroxymethylation in these features showed nonlinear correlation with expression in the median tier. This trend persisted despite the significant decrease of $5 \mathrm{hmC}$ in cancer observed in exonic and DHS-proximal regions and may indicate altered functionality of $5 \mathrm{hmC}$ marks in prostate cancer.

The same correlation was also performed for RWPE-1 hMeDIP-seq data (Additional file 1: Table S5), where intergenic, genic, and DHS-proximal trends from hMeSeal-seq were strongly corroborated (Additional file 1: Figure S8).

\section{Absence, gain, and retention of $5 \mathrm{hmC}$ differs significantly by genomic region between normal and cancer cell lines} We examined absolute peak regions where $5 \mathrm{hmC}$ marks detected in RWPE-1 either overlapped with peak regions detected as having $5 \mathrm{mC}$ marks in 22Rv1 (5hmC "absence") or 5hmC marks in 22Rv1 (5hmC "retention"), as well as peaks where $5 \mathrm{mC}$ marks in RWPE-1 overlapped with $5 \mathrm{hmC}$ marks in 22Rv1 (5hmC "gain"). We then tested these peak regions, stratified by genomic location, to determine genomic features where the frequency of occurrence of these overlapping peaks could not be explained by either random change in RWPE- 1 epigenetic marks or by random distribution of 22Rv1 marks within each genomic feature (Additional file 1: Table S6).

Peaks located within exonic, promoter, or RWPE-1 DHS genomic features exhibited greater $5 \mathrm{hmC}$ depletion than expected from the proportion of global RWPE- $15 \mathrm{hmC}$ or 22Rv1 5mC; however, they did not significantly differ from expected values for random $5 \mathrm{hmC}$ retention or gain in cancer (Table 1). In other words, peaks within these features were far more likely to exhibit absence of $5 \mathrm{hmC}$ in cancer than to gain or retain it (Additional file 1: Table S7), suggesting that loss of $5 \mathrm{hmC}$ within these specific 
Table 1 Locus-specific hydroxymethylation changes in cancer. $5 \mathrm{hmC}$ changes were described by the overlap of $5 \mathrm{mC}$ and/or $5 \mathrm{hmC}$ marks between cell lines. Overlapping $5 \mathrm{mC}$ or $5 \mathrm{hmC}$ marks were compared to the overall distribution of each mark within each cell line to determine significant differences

\begin{tabular}{|c|c|c|c|c|c|c|c|c|c|}
\hline \multirow{2}{*}{$\begin{array}{l}\text { Differential } \\
5 \mathrm{mC} / 5 \mathrm{hmC} \\
\text { status in PCa }\end{array}$} & \multirow{2}{*}{$\begin{array}{l}\text { Prostate cell } \\
\text { type (normal } \\
\text { vs. cancer) }\end{array}$} & \multirow{2}{*}{$\begin{array}{l}\text { Overlapping } \\
\text { methylation } \\
\text { marks }\end{array}$} & \multicolumn{7}{|c|}{ Genomic region } \\
\hline & & & Intergenic & Genic & Exonic & Intronic & Promoter & CpG Island & $\begin{array}{l}\text { RWPE-1 DNasel } \\
\text { hypersensitive sites }\end{array}$ \\
\hline \multirow[t]{2}{*}{ 5hmC "Absent" } & RWPE-1 & $5 \mathrm{hmC}$ & 0.051 & 0.666 & $<0.0001^{*, * *}$ & $0.005^{*, * * *}$ & $<0.0001^{* * * *}$ & $<0.0001^{* * * *}$ & $<0.0001^{* * * *}$ \\
\hline & 22Rv1 & $5 \mathrm{mC}$ & $<0.0001^{*}$ & 0.311 & $0.002^{* * * *}$ & $0.001^{* * * * *}$ & $<0.0001^{* * *}$ & $<0.0001^{* * * *}$ & $<0.0001^{* * *}$ \\
\hline \multirow[t]{2}{*}{$5 \mathrm{hmC}$ "Retained" } & RWPE-1 & $5 \mathrm{hmC}$ & 0.302 & 0.01 & 0.039 & 0.169 & 0.487 & 0.593 & 0.067 \\
\hline & $22 \mathrm{Rv} 1$ & $5 \mathrm{hmC}$ & 0.013 & 0.547 & 0.452 & 0.834 & 0.115 & $0.001^{*}$ & 0.944 \\
\hline \multirow[t]{2}{*}{$5 \mathrm{hmC}$ "Gained" } & RWPE-1 & $5 \mathrm{mC}$ & $0.003^{*, * * *}$ & 0.975 & 0.175 & 0.834 & 0.5 & $<0.0001^{*, * *}$ & $0.007^{*}$ \\
\hline & $22 \mathrm{Rv} 1$ & $5 \mathrm{hmC}$ & $<0.0001^{* * * * *}$ & 0.026 & 0.025 & 0.538 & 0.71 & $<0.0001^{* * * *}$ & 0.511 \\
\hline
\end{tabular}

*Significant $p$ values as determined by the chi-square test with Bonferroni correction for multiple testing applied; **5hmC is significantly more likely to be absent, gained, or retained within the given region than expected; ${ }^{* *} 5 \mathrm{hmC}$ is significantly less likely to be absent, gained, or retained within the given region than expected

features may be especially important in tumorigenesis. In contrast, intergenic regions in cancer showed far less gain of $5 \mathrm{hmC}$ than expected, despite being the only region in which proportional $5 \mathrm{hmC}$ absence in $22 \mathrm{Rv} 1$ was nonsignificant. Intriguingly, both absence and gain of $5 \mathrm{hmC}$ was greater than expected within CGIs, while intronic regions showed significantly less gain of $5 \mathrm{hmC}$ than expected. Unlike all other features tested, however, the proportions of peak regions showing $5 \mathrm{hmC}$ absence, gain, or retention in intronic regions did not significantly differ from each other. These findings further indicate that changes in $5 \mathrm{hmC}$ regulation and functionality in cancer differ between specific genomic features. However, despite the significant changes in $5 \mathrm{hmC}$ absence in both intronic and exonic regions, genic changes (comprised of the sum of intronic, exonic, and UTR regions) in $5 \mathrm{hmC}$ status were not found to be significantly different from random distribution in any category.

\section{Biological pathways regulated by $5 \mathrm{mC}$ and $5 \mathrm{hmC}$ marks differ significantly between normal prostate and prostate cancer}

We performed pathway analysis on genes enriched in either $5 \mathrm{mC}$ or $5 \mathrm{hmC}$ differential marks within each tier of expression and genomic feature. To identify differential gene regulation by each mark in normal versus cancer, we compared the most significant and specific annotation terms (defined by grouping similar enriched pathways using the MCODE clustering algorithm and manually labeling the groups), identified via the Genomic Regions Enrichment of Annotation Tool (GREAT), between cell lines (hypergeometric test over regions; false discovery rate $($ FDR $)<0.05)$. Individual enrichment maps were generated for each feature and expression tier within each cell line prior to pathway comparison (Fig. 3a).

Pathway enrichment for differentially methylated genes was primarily observed within CGIs for 22Rv1, where multiple genes exhibiting CGI methylation and functionally enriched for annotations related to the regulation of cell proliferation, adenylate cyclase activity, and lyase activity displayed lowered expression in cancer compared to normal (Additional file 1: Figure S9A). In contrast, annotations for differentially methylated genes in exonic regions were primarily enriched for core cellular functions in both cell lines (Additional file 1: Figure S9BS9C). Similarly, although differential intronic methylation patterns showed enrichment for core pathways in both cell lines (Additional file 1: Figure S10A-S10B), most genes did not exhibit concordant differential expression tiers from the normal to the cancer cell line.

Contrary to our expectations, $5 \mathrm{hmC}$ expression pathway trends paralleled $5 \mathrm{mC}$ expression trends within CGI and exonic regions in cancer. While hydroxymethylated genes in CGIs remained unenriched for relevant pathways in RWPE-1 cells (Additional file 1: Figure S11A), annotations related to Akt, Wnt, and mTor signaling, as well as inhibitory p53 feedback loops, were abundant within highly expressed genes exhibiting CGI hydroxymethylation in 22Rv1 (Additional file 1: Figure S11B). However, all genes involved were also highly expressed in normal cells.

Exonic hydroxymethylation terms, in contrast, were not significantly enriched in $22 \mathrm{Rv} 1$ (data not shown) but displayed enrichment within the highest tier of expression for a diverse range of pathways (including housekeeping genes expressed across 19 normal tissues, genes able to induce cell death, and vitamin D metabolism) within RWPE-1 (Fig. 3b). Furthermore, many of these genes showed reduced expression in our cancer cell line, which may indicate a role for exonic $5 \mathrm{hmC}$ in the normal regulation of core cellular functions.

Similar to intronic $5 \mathrm{mC}$-expression profiling patterns, hydroxymethylated intronic regions in RWPE-1 showed few significant pathway annotations within the lowest tier of expression (Fig. 3c), unlike 22Rv1 (Fig. 3d). 


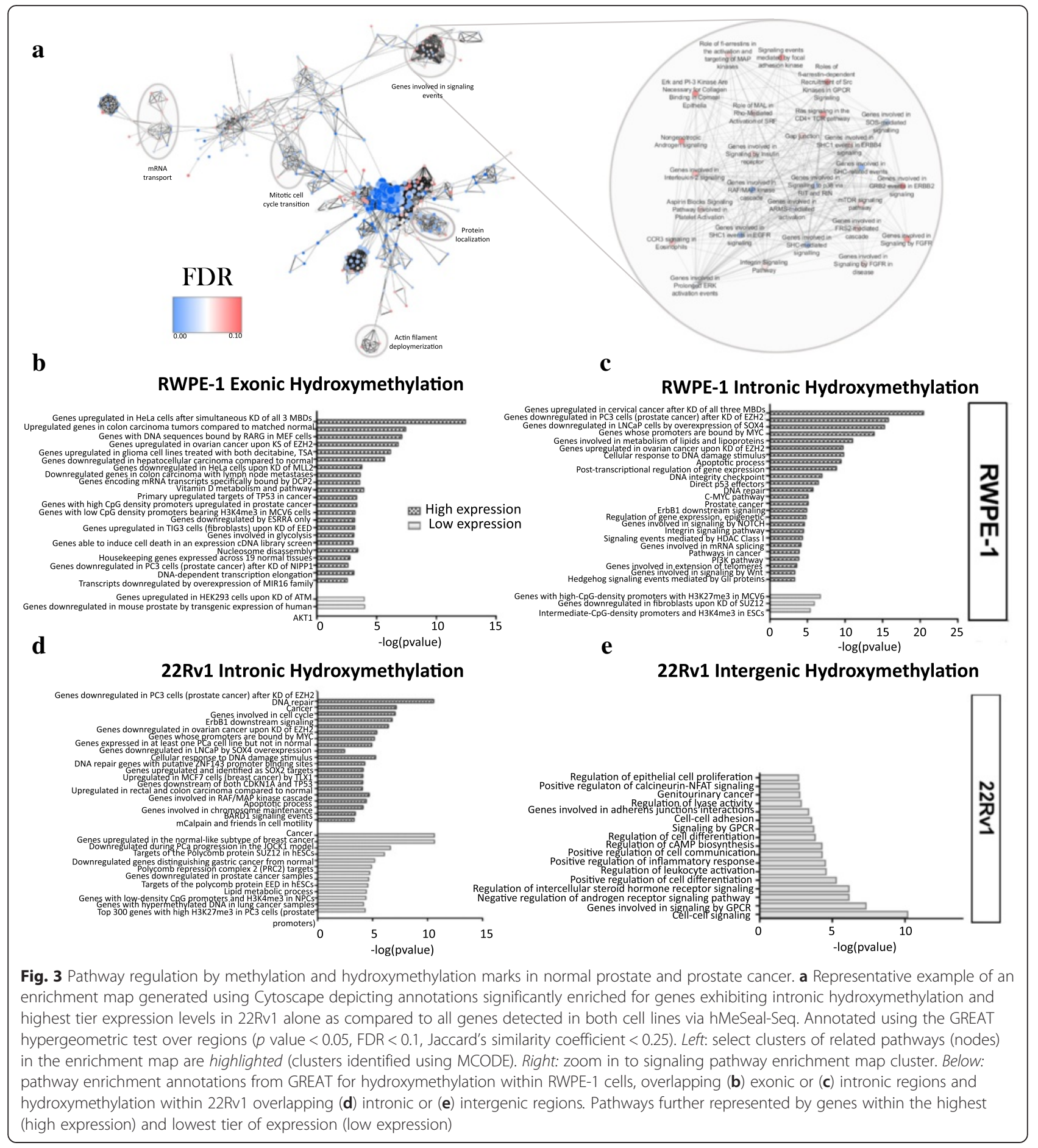

However, extensive enrichment was observed within the highest expression tier for pathways related to DNA damage repair, integrity checkpoints, apoptosis, and direct p53 effectors (Fig. 3c). Interestingly, annotations related to prostate cancer, the c-Myc pathway, and telomere extension were also observed within the normal cell line within the highest expression tier. As the RWPE-1 cell line has been immortalized through the use of human papillomavirus, these pathways may be indicative of aberrations incurred from the immortalization process. In cancer, significant pathway enrichment was seen in both low and high expression tiers, with polycomb repressive complex 2 (PRC2) targets and genes known to be downregulated in $\mathrm{PCa}$ enriched in the lowest expression tier while pathways related to cell motility, BARD1 and ErbB1 signaling, and 
genes known to be enriched in PCa specifically were observed in the highest expression tier (Fig. 3d). Interestingly, DNA repair and apoptotic pathway enrichment was also seen within this tier in cancer, although most genes involved were expressed at the same level in both normal and PCa cells.

Perhaps most intriguingly, intergenic regions exhibited pathway enrichment within the lowest tier of expression unique to $22 \mathrm{Rv} 1$ cells. Strikingly, many of these pathways were associated with tumorigenic functions (Fig. 3e), suggesting a putative role for intergenic hydroxymethylation in the downregulation of inflammation and cellular adhesion and the upregulation of epithelial cell proliferation. Of particular interest, however, was the presence of $5 \mathrm{hmC}$ enrichment in pathways governing the negative regulation of androgen signaling. Furthermore, differentially hydroxymethylated genes within this pathway, including the PCa-inhibitory genes secreted fizzled-related protein 1 (SFRP1) and Dab, mitogen-responsive phosphoprotein homolog 2 (DAB2), exhibited significantly lower expression in cancer than in the normal cell line. In sharp contrast, RWPE-1 showed few significant functional annotations for low-tier intergenic hydroxymethylation marks (Additional file 1: Figure S12). Overall, exonic and intergenic regions showed the most striking differential pathway enrichment between normal and cancer cells for both $5 \mathrm{mC}$ and $5 \mathrm{hmC}$ marks. Differentially hydroxymethylated genes from high-level exonic pathways in RWPE-1 and low-tier intergenic pathways in 22Rv1 exhibited large differences in expression between cell lines, potentially indicating a critical role for aberrations in exonic and intergenic patterning of $5 \mathrm{mC}$ and $5 \mathrm{hmC}$ marks in cancer.

\section{Locus-specific $5 \mathrm{mC}$ and $5 \mathrm{hmC}$ marks share a novel} cooperative role in the regulation of functional pathways We compared biological pathway annotations for genes enriched in both $5 \mathrm{mC}$ and $5 \mathrm{hmC}$ within each cell line by expression tier and genomic feature in order to determine whether or not intercellular differences observed in the type of epigenetic modifications resulted in significant differences in pathway regulation (Additional file 1). Surprisingly, we found that a significantly higher proportion of pathways enriched for both marks in either cell line showed intracellular co-enrichment of $5 \mathrm{hmC}$ and $5 \mathrm{mC}$ within the same expression tier on the same gene $(p<0.0001)$. While promoter and CGI methylation and hydroxymethylation marks governed completely different pathways, genes with intronic $5 \mathrm{hmC}$ marks exhibited strong $5 \mathrm{mC}$ and $5 \mathrm{hmC}$ co-enrichment for specific cellular functions in both cell lines (Fig. 4a). Furthermore, while gene-specific methylation and exonic hydroxymethylation were observed to co-regulate pathways related to basic cellular functionalities such as ATP binding and hydrolase activity in RWPE-1 (Fig. 4b), this co-regulation was entirely lost in 22Rv1, mirroring the loss of exonic $5 \mathrm{hmC}$ marks in cancer. Intriguingly, co-occurrence of $5 \mathrm{mC}$ with intergenic $5 \mathrm{hmC}$ epigenetic marks was observed in 22Rv1, but not in RWPE-1, coinciding with high-level functional annotations in the lowest tier of expression including signaling, regulation of cellular proliferation and cAMP biosynthesis regulation in cancer (Fig. 4c).

Additionally, we found that most hydroxymethylated intronic pathways showing concomitant $5 \mathrm{mC}$ enrichment in cancer were related to basic cellular functions, such as binding, protein localization, and cytoskeletal organization, within each expression tier (Fig. 4d). This observation contrasts sharply with hydroxymethylated intronic co-enrichment pathways in RWPE-1, where basic processes predominated in the middle and lowest expression tiers (Fig. 4e). However, annotations for genes exhibiting both marks within the highest tier of expression were enriched for multiple highly specific processes, including pathways related to the electron transport chain as well as binding of both NF- $\mathrm{kB}$ and p53. This data provides novel evidence for the highly locus- and gene-specific dysregulation of $5 \mathrm{hmC}$ in governing oncogenesis.

\section{Genes involved in key differential biological processes are detectable via hMeSeal-qPCR}

We chose representative genes for validation which were localized to hydroxymethylated regions detected by both hMeSeal-seq and hMeDIP-seq in RWPE-1, were not hydroxymethylated in 22Rv1, and overlapped RWPE-1 DHSs (Additional file 1: Table S8). Selected genes were not methylated in either cell line (Additional file 1: Figure S13). Cullin 2 (CUL2) bore methylation marks overlapping hydroxymethylation peaks in RWPE-1, but not in 22Rv1; in contrast, RasGEF domain family member 1a (RASGEF1a) and histidine triad nucleotidebinding protein 1 (HINT1) were methylated in the same region in 22Rv1 (exhibiting $5 \mathrm{hmC}$ "loss" in cancer while not being methylated in RWPE-1). RASGEF1a and CUL2 were located in intronic regions, while HINT1 refers to an intergenic $5 \mathrm{hmC}$ peak located most closely to the TSS of HINT1. Lastly, all genes chosen possessed robust and clear $5 \mathrm{hmC}$ enrichment peaks within the overlapping regions, with corresponding absent or low peaks in input samples (Fig. 5a).

In order to validate the specificity of our sequencing data and quantify the relative $5 \mathrm{hmC}$ levels, we performed qPCR with standard curve protocol following hMeSeal enrichment of genomic RWPE-1 and 22Rv1 DNA. We used primers flanking the identified enrichment peaks for CUL2, RASGEF1a, and HINT1, as well 


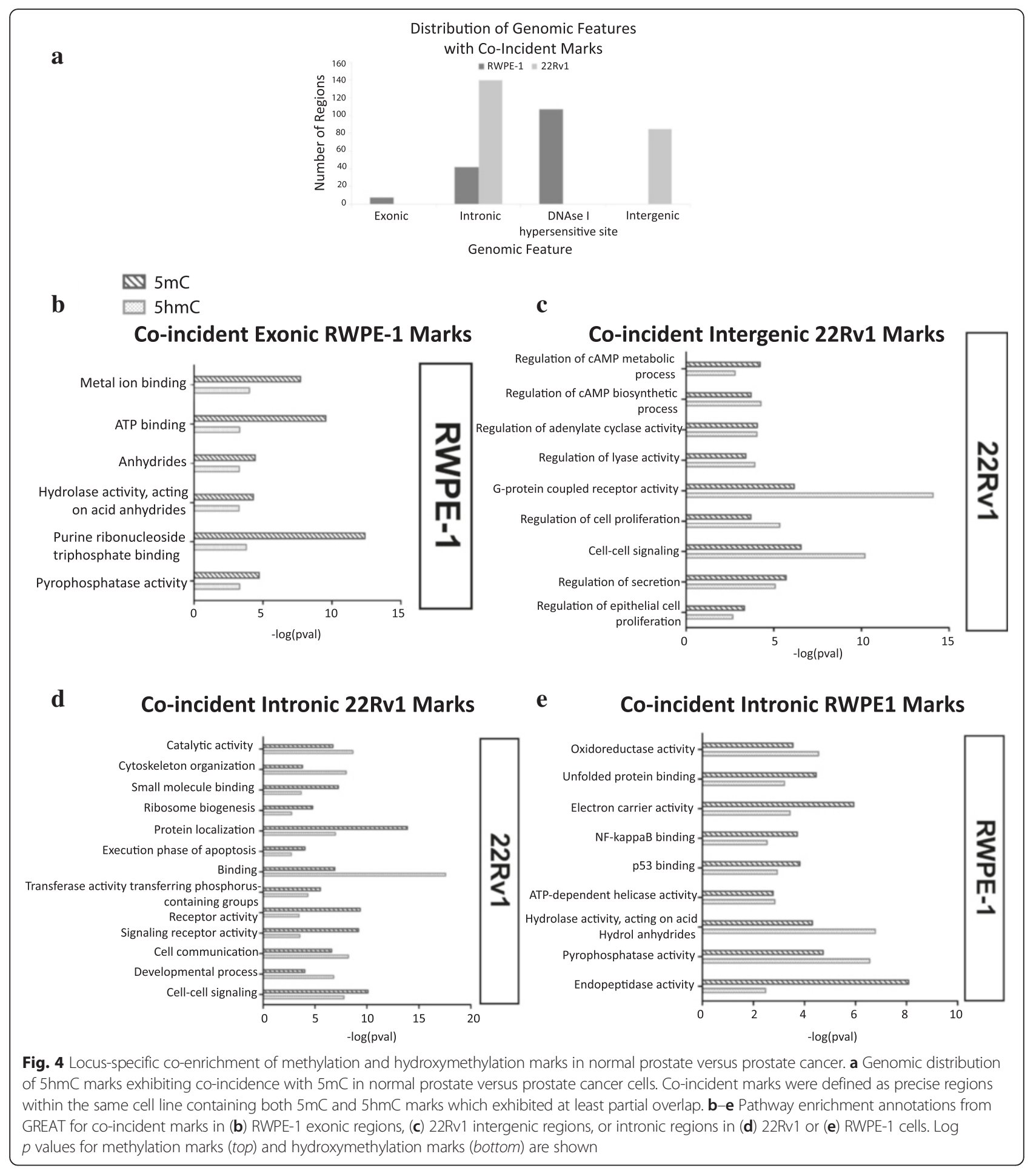

as the negative control gene homeobox D8 (HOXD8). HOXD 8 was found to be strongly methylated in RWPE1 (Additional file 1: Figure S14) and was not hydroxymethylated in either cell line. Negative control capture reactions lacking UDP-azide-glucose followed by qPCR were performed simultaneously across three technical replicates. Enrichment of HOXD8 was not detected via
qPCR, confirming the specificity of our capture reaction. CUL2, RASGEF1a, and HINT1 positive and negative control qPCR reactions were normalized to HOXD8 negative control using the $\Delta \Delta \mathrm{C}_{\mathrm{t}}$ method, obtaining fold enrichment values of $2.73 \times, 3.20 \times$, and $4.39 \times$, respectively (Fig. 5b), while enrichment was not detectable in 22Rv1 (Fig. 5c). These findings validate our hMeSeal-seq 


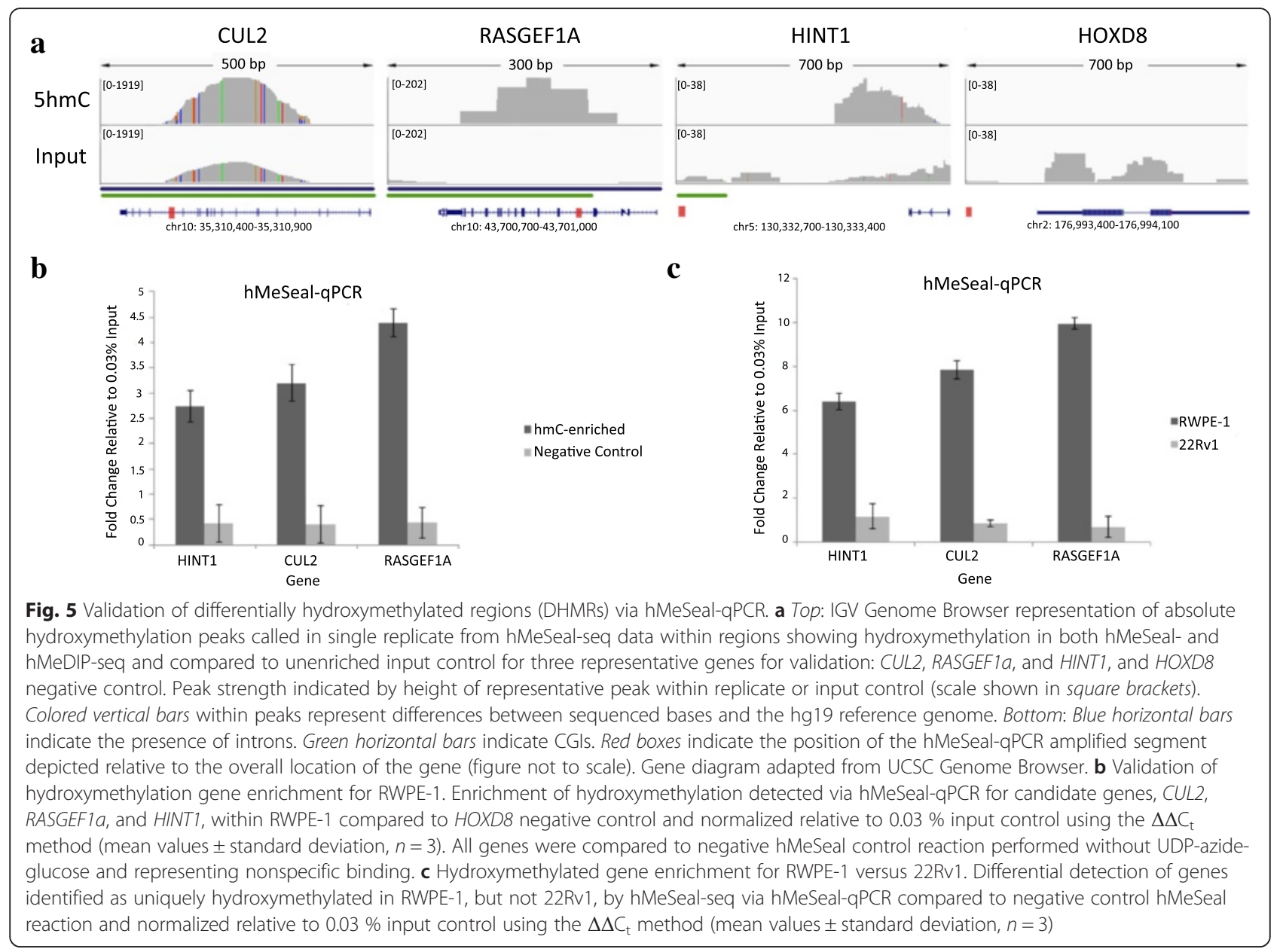

results and show that locus-specific detection of $5 \mathrm{hmC}$ enrichment is possible in the prostate and prostate cancer cell lines.

\section{Discussion}

Recent studies have provided direct evidence supporting a key role for $5 \mathrm{hmC}$ in regulating transcription, both through the structural influence of locus-specific $5 \mathrm{hmC}$ hemimodification on transcription factor binding [38] and the active recruitment of $5 \mathrm{hmC}$ "readers" regulating transcription as described in murine retinal development [39]. In hematological cancers such as acute myeloid leukemia, locus-specific loss of $5 \mathrm{hmC}$ has been negatively correlated with gene expression in intronic, genic, promoter, and distal regulatory regions [40]. However, previous studies in solid tumors have focused primarily on mapping genome-wide loss of hydroxymethylation independently of genomic features [7, 9, 21]. Exceptions include pancreatic cancer, in which a positive association was found between promoter or gene body $5 \mathrm{hmC}$ enrichment and gene expression in both normal and cancer cells [30], and in colon cancer, in which promoter hydroxymethylation in normal cell lines was found to render them resistant to oncogenic methylation gain [13], and also melanoma, in which enrichment of oncogenic functions was found in genes losing $5 \mathrm{hmC}$ marks and gaining $5 \mathrm{mC}$ marks in cancer [41].

In prostate cancer, $5 \mathrm{hmC}$ has been found to be both globally depleted and inversely correlated with cell proliferation [7, 37] and has recently been implicated as an inhibitory mark downregulating gene expression in metastatic PCa cell line models [42], in direct contrast to our findings. As the distribution of hydroxymethylation marks differs significantly between tissue types [9], this discrepancy may be reflective of differences between $\mathrm{PCa}$ models or may even be indicative of differential genomic feature-specific effects of $5 \mathrm{hmC}$ modification. To date, the locus-specific role of $5 \mathrm{hmC}$ modification in governing gene expression and functionality has not been identified in PCa. Furthermore, while oncogenic pathway enrichment has been found in regions exhibiting $5 \mathrm{hmC}$ gain in pancreatic cancer [37], our data did not reveal significant pathway enrichment for similar genes in our cell lines. This may be due to significant 
global loss of $5 \mathrm{hmC}$ marks in cell culture further impairing detection of hydroxymethylated regions in our $\mathrm{PCa}$ cell line [35] or an example of a tissue-specific $5 \mathrm{hmC}$ profile.

One important caveat of our cell line model is the rapid global loss of $5 \mathrm{hmC}$ observed by Nestor et al. in cell lines almost immediately upon culture and passaging [35]. High-passage mouse embryonic fibroblast cell lines and primary $\mathrm{T}$ cell cultures were found to lose $5 \mathrm{hmC}$ to such a degree that clear hydroxymethylation patterning was lost from the tissues or primary cells of origin. Loss of $5 \mathrm{mC}$ in culture, however, was much milder and allowed for the retention of patterning. Although global levels of $5 \mathrm{hmC}$ in our cell lines were low, it should be noted that our validation of hMeSeal-seq data via both genome-wide hMeDIP-seq and locus-specific hMeSealqPCR contraindicates the loss of clear $5 \mathrm{hmC}$ patterning within our cell line models.

The overall distribution of $5 \mathrm{mC}$ and $5 \mathrm{hmC}$ marks within each cell line showed an absolute methylation peak increase and hydroxymethylation peak decrease for all genomic features in cancer. However, the lack of significant intronic $5 \mathrm{hmC}$ difference between cell lines, coupled with our observation that intronic regions are the most likely to gain $5 \mathrm{hmC}$ in $\mathrm{PCa}$, may indicate that intronic hydroxymethylation is both tightly regulated and potentially critical for basic cellular function. Similarly, the proportional gain of intergenic $5 \mathrm{hmC}$ and its status as the most likely feature to retain $5 \mathrm{hmC}$ in cancer may indicate either a lack of importance for intergenic $5 \mathrm{hmC}$ marks or, conversely, a key regulatory function in oncogenic transformation. However, the strong negative correlation between intergenic hydroxymethylation and gene expression in both cell lines, in addition to the significant pathway enrichment in genes bearing intergenic $5 \mathrm{hmC}$ and in the lowest tier of expression for terms related to androgen receptor regulation, inflammation, and cellular adhesion, provides strong support for the latter hypothesis. Our findings may indicate novel biological roles for intergenic $5 \mathrm{hmC}$ modifications located outside of regulatory regions and intron-specific $5 \mathrm{hmC}$ marks gained in cancer. Since many different factors can contribute to results from pathway-based analysis, further functional validation of implicated candidate genes will be performed using in vitro cell lines and animal models in order to verify these findings.

In contrast, although CGIs were found to be generally depleted for $5 \mathrm{hmC}$ marks in both of our cell lines, to such an extent that expression patterning was entirely lost in cancer, the extent of $5 \mathrm{hmC}$ depletion and gain within these features suggests that specific changes in CGI hydroxymethylation may be especially important for not only downregulation of genes in PCa but their upregulation as well. Interestingly, despite the significant exonic absence and intronic retention of $5 \mathrm{hmC}$ in cancer, genic regions exhibit no significant differences in $5 \mathrm{hmC}$ from random changes in either cell line, suggesting that the level of $5 \mathrm{hmC}$ retention in introns matches the magnitude of $5 \mathrm{hmC}$ depletion in exonic regions. Therefore, although many studies examine genic $5 \mathrm{hmC}$ alterations at a global level in development and disease, the observed distinct function of $5 \mathrm{hmC}$ within exons and introns in our samples underscores the need for further functional studies of $5 \mathrm{hmC}$ to be performed in a genomic feature-specific manner.

We found that functional mark distribution clearly suggested a distinct biological role for $5 \mathrm{mC}$ and $5 \mathrm{hmC}$ within our cell lines. Our analysis revealed several major biological pathways exhibiting differential epigenetic regulation in normal and cancer cell lines. Within 22Rv1 CGIs, 5hmC was positively correlated with genes involved in signaling processes promoting cell proliferation and migration and downregulating apoptotic p53 signaling, while $5 \mathrm{mC}$ was simultaneously negatively correlated with expression of genes regulating cyclic AMP (cAMP) generation. These observations reflect the contrasting relationships between CGI methylation or hydroxymethylation and expression.

Additionally, we observed distinct biological roles for $5 \mathrm{mC}$ and $5 \mathrm{hmC}$ marks between cell lines. Intronic hydroxymethylation was not significantly lost in our cancer cell line compared to normal, indicating that $5 \mathrm{hmC}$ may undergo specific redistribution in tumorigenesis. For example, while RWPE-1 intronic hydroxymethylation appears to positively regulate genes related to calpain I (uCalpain) activity in cell adhesion, 22Rv1 intronic $5 \mathrm{hmC}$ is enriched for pathways related to calpain II (mCalpain) involvement in cell motility. Intriguingly, almost all RWPE-1 hydroxymethylated genes involved in the tumor necrosis factor pathway exhibit lowered expression in 22Rv1, indicating a critical role for intronic $5 \mathrm{hmC}$ in governing this pathway. Due to the potentially confounding effects of immortalization in our cell lines, future functional analysis will be performed in tissue samples and in vivo models in order to further investigate the differential functionality implied by pathway analysis.

Many of the annotations observed for genes coincident for $5 \mathrm{hmC}$ and $5 \mathrm{mC}$ marks in either cell line are related to basic cellular functions, which are often broad categories involving many genes [41, 43]. However, we also observed striking differences in both mark distribution and annotations between cell lines and genomic features with specialized pathways that are more likely to be indicative of true functional differences in epigenetic regulation.

Co-incidence of $5 \mathrm{mC}$ with exonic $5 \mathrm{hmC}$ was lost in 22Rv1 compared to normal, along with overall exonic 
$5 \mathrm{hmC}$ levels, representing a loss of co-incident marks in basic cellular pathways observed in RWPE-1. Intriguingly, this observation stands almost in direct contrast to intronic $5 \mathrm{hmC}$ and $5 \mathrm{mC}$ mark profiling in cancer, where intronic retention of both marks was seen for terms related to basic cellular functionality. This may possibly indicate their more essential role in basic cell survival than exonic modifications. Alternatively, it may also suggest that the genes involved in the cellular processes enriched for $5 \mathrm{mC}$ co-incidence with exonic $5 \mathrm{hmC}$ are more likely to exert functions inhibitory to tumor growth than those in intronic regions. Considering that overall $5 \mathrm{hmC}$ levels within introns are still lost in 22Rv1, an examination of noncoding RNA expression from intronic regions exhibiting differential epigenetic regulation in cancer might reveal the function of specific mark retention or gain in PCa.

The proportional increase of intergenic $5 \mathrm{hmC}$ in cancer, coupled with its robust correlation to low gene expression, may indicate a novel cooperative role for locus-specific $5 \mathrm{hmC}$ and $5 \mathrm{mC}$ in the regulation of tumor-suppressive functions in both normal and aberrantly modified PCa cell lines. Furthermore, this data suggests not only the precise dysregulation of nonessential $5 \mathrm{hmC}$ in intronic pathways related to tumorsuppressive functions in cancer but also that locusspecific intergenic gain of $5 \mathrm{hmC}$ may be used by tumor cells in conjunction with aberrant methylation elsewhere on the gene to actively suppress antitumor function.

In accordance with our novel findings regarding the role of intronic and intergenic hydroxymethylation in $\mathrm{PCa}$, we chose two intronic genes-RASGEF1a and CUL2-and one intergenic gene-HINT1-for validation studies based primarily on the robustness of concordance between hMeDIP and hMeSEAL peaks, as well as lack of methylation and functionality in cancer.

Overexpression of RASGEF1a, an activator of the RAS family of oncogenes promoting cellular migration, is linked to oncogenesis in cholangiocarcinoma [44, 45]. The hydroxymethylation peak detected for RASGEF1a was located at the exon-intron boundary, a region known to exhibit neuronal $5 \mathrm{hmC}$ enrichment in the literature [11]. In normal cells, HINT1 exerts tumorsuppressive effects through inhibition of the Wnt pathway and the promotion of both apoptosis and p53 expression and is known to undergo transcriptional silencing via promoter hypermethylation in colon and non-small cell lung cancer [46, 47]. Despite exhibiting the weakest peak of all three genes chosen for validation, the HINT1-proximal $5 \mathrm{hmC}$ peak was still robustly detected by hMeSeal-qPCR, confirming that intergenic $5 \mathrm{hmC}$ marks can also be reliably detected using our whole-genome sequencing strategy. Finally, CUL2 was chosen as a representative validation gene due to the strength of its intronic hMeSeal-seq peak and its function as a component of the tumor-suppressive Von Hippel-Lindau complex, inhibiting uncontrolled angiogenesis via ubiquitination and degradation of hypoxiainducible factor 1 alpha (HIF1 $\alpha)$ [48]. As all three genes were able to be detected specifically in the normal cell line, we have verified the ability of our hMeSeal-Seq technique to reliably and specifically detect differentially distributed and weakly hydroxymethylated genes.

Since our strategy for selecting representative candidates was successful, we propose that further $5 \mathrm{hmC}$ candidates be selected in this manner. Key candidate genes involved in the regulation of critical oncogenic processes will be identified from region-specific central biological pathways exhibiting differential $5 \mathrm{hmC}$ modification based on hMeSeal-seq peak strength, function, annotation significance and relevance, putative association with TET enzymes, and differential gene expression between normal and cancer cell lines. Ultimately, these genes will be validated in primary tissue samples from both normal and early-stage cancer, as we expect that specific changes in $5 \mathrm{hmC}$ marks may be an early event in tumorigenesis.

\section{Conclusions}

We demonstrate the highly locus-specific correlation of hydroxymethylation with gene expression in normal prostate cells, with a loss of robustness for correlation with positive expression in intergenic regions as well as a novel observation of increased robustness in negative expression correlation. Our findings indicate that loss of $5 \mathrm{hmC}$ in exonic, intronic, and CGI genomic regions, coupled with novel proportional hydroxymethylation gain in intergenic regions, may constitute important mechanisms of transcriptional repression in cancer development. Simultaneously, integrative pathway analysis correlating $5 \mathrm{hmC}$ to $5 \mathrm{mC}$ mark distribution, stratified by genomic feature, reveals their cooperative role in governing key biological pathways implicated in $5 \mathrm{hmC}$ directed regulation of tumorigenesis. The selection of candidate genes exhibiting differential expression and epigenetic regulation from these functional pathways may provide novel avenues for the investigation of new and pre-existing biomarkers of cancer development.

Our locus-specific integrative analysis of hydroxymethylation marks is the first study of its kind to be performed in prostate cancer. Ultimately, our characterization of key cellular pathways exhibiting dynamic enrichment alterations for $5 \mathrm{hmC}$ or $5 \mathrm{mC}$ marks may not only allow us to identify novel biomarkers of disease but may also lead to the discovery of potential therapeutic target genes in PCa.

\section{Methods}

Cell culture and DNA extraction

Normal human prostate epithelial cell line, RWPE-1, was obtained from the American Type Culture 
Collection (ATCC). Human prostate cancer cell line, 22Rv1, was provided by Dr. E. Diamandis (Mount Sinai Hospital). RWPE-1 cells were cultured with Keratinocyte serum-free medium (K-SFM) (Invitrogen) supplemented with $0.05 \mathrm{mg} / \mathrm{ml}$ bovine pituitary extract (BPE) and $5 \mathrm{ng} / \mathrm{ml}$ human recombinant epidermal growth factor (EGF). 22Rv1 cells were cultured with RPMI 1640 (Mount Sinai Hospital) with $10 \%$ fetal bovine serum (FBS). All cells were cultured as a monolayer and maintained in a humidified incubator at $37{ }^{\circ} \mathrm{C}$ with $5 \% \mathrm{CO}_{2}$. Genomic DNA was extracted from cells after trypsinization, using the QIAamp DNA Mini Kit (Qiagen) following the protocol provided.

\section{DNA methylation enrichment and next-generation se- quencing: MBD-seq}

Genomic DNA extracted from RWPE-1 and 22Rv1 cells was sonicated into approximately 100-300 bp fragments using a Vibracell Disrupter (SONICS). Sheared genomic DNA $(4 \mu \mathrm{g})$ was incubated with methyl-CpG binding domain 2 (MBD2) protein and magnetic streptavidin beads provided by the MethylMiner Kit (Invitrogen). Bound DNA fragments were eluted with the highest concentration of $\mathrm{NaCl}$ buffer $(2000 \mathrm{mM})$. Bound, unbound, and input DNA fragments were precipitated using MinElute PCR Purification Kit (Qiagen) with the final elution in UltraPure Distilled Water (Invitrogen). Bound and input DNA was quantified using Qubit 2.0 Fluorometer (Invitrogen). Bound, MBD2-enriched DNA (20 ng), and input samples (non-enriched) from 22Rv1 and RWPE-1 cells were submitted in triplicate biological replicates for library preparation (NEBNext ${ }^{\circ}$ ChIP-Seq Library Prep Reagent Set for Illumina) and highthroughput sequencing using the HiSeq 2000 and 2500 (Illumina) at The Centre for Applied Genomics (The Hospital for Sick Children). Each library generated approximately 50 million paired-end reads with $5 \times$ coverage of CpGs.

\section{DNA hydroxymethylation enrichment by glucosylation and next-generation sequencing: hMeSeal-seq}

Genomic DNA extracted from RWPE-1 and 22Rv1 cells was sonicated into approximately 100-300 bp fragments using a Q125 sonicator (QSonica). Sheared genomic DNA $(10 \mu \mathrm{g})$ was specifically glucosylated at $5 \mathrm{hmC}$ by T4-phage $\beta$-glucosyltransferase and incubated with biotin and streptavidin beads provided by the Hydroxymethyl Collector Kit (Active Motif). The final elution of bound, unbound, and input DNA fragments were precipitated according to the protocol provided with the final elution in UltraPure Distilled Water (Invitrogen). Bound and input DNA was quantified using Qubit 2.0 Fluorometer (Invitrogen). Each replicate of $5 \mathrm{hmC}$-enriched sample was prepared by pooling up to four hMeSeal reactions. Following, bound/glucosylated 5hmC-enriched DNA (15 ng) and input samples (non-enriched) from RWPE-1 and 22Rv1 cells were submitted in triplicates for library preparation (NEBNext ${ }^{\circ}$ ChIP-Seq Library Prep Reagent Set for Illumina) and high-throughput sequencing using the HiSeq 2500 (Illumina) at The Centre for Applied Genomics (The Hospital for Sick Children). Each library generated approximately 50 million paired-end reads, with $10 \times$ coverage of CpGs.

\section{DNA hydroxymethylation enrichment by immunoprecipitation and next-generation sequencing: hMeDIP-seq}

RWPE-1 genomic DNA from a separate biological replicate was submitted to Arraystar Inc. (Rockville, Maryland) where hMeDIP enrichment and NGS processing were performed. Genomic DNA was sonicated into approximately 200-600 bp fragments with a Bioruptor sonicator (Diagenode). Sheared genomic DNA (800 ng) was end-repaired, A-tailed, and ligated to single-end adapters following the standard Illumina genomic DNA protocol. Agarose size-selection was used to remove unligated adapters. Adaptor-ligated DNA was heatdenatured at $94{ }^{\circ} \mathrm{C}$ for $10 \mathrm{~min}$ and rapidly cooled on ice. Following, the denatured DNA was immunoprecipitated with $1 \mu \mathrm{L}$ mouse monoclonal anti-5-hmC antibody (Diagenode) overnight at $4{ }^{\circ} \mathrm{C}$, with rocking agitation in $400 \mu \mathrm{L}$ immunoprecipitation (IP) buffer $(0.5 \%$ BSA in PBS). Five immunoprecipitation washes were performed with ice-cold IP buffer. A nonspecific mouse IgG immunoprecipitation was performed as a negative control. Washed beads were resuspended in TE buffer (with $0.25 \%$ SDS and $0.25 \mathrm{mg} / \mathrm{mL}$ proteinase $\mathrm{K}$ ) for $2 \mathrm{~h}$ at $65{ }^{\circ} \mathrm{C}$ and then cooled to room temperature. Bound/ immunoprecipitated and unbound DNA fragments were purified using MinElute columns (Qiagen) and eluted in $16 \mu \mathrm{L}$ of elution buffer (Qiagen). Immunoprecipitated fragments $(5 \mu \mathrm{L})$ were subjected to 14 cycles of PCR using single-end PCR primers (Illumina). The resulting reactions were purified with MinElute columns (Qiagen), after which a final size selection (300-700 bp) was performed by electrophoresis in $2 \%$ agarose. Libraries were quality controlled by Agilent 2100 Bioanalyzer. An aliquot of each library was diluted in elution buffer (Qiagen) to $5 \mathrm{ng} / \mu \mathrm{L}$, and $1 \mu \mathrm{L}$ was used in real-time $\mathrm{PCR}$ reactions to confirm the enrichment for hydroxymethylated region. The library was denatured with $0.1 \mathrm{M}$ $\mathrm{NaOH}$ to generate single-stranded DNA molecules, and loaded onto channels of the flow cell (8 pM), and amplified in situ using TruSeq Rapid SR Cluster Kit (\#GD402-4001, Illumina). High-throughput sequencing was performed on the HiSeq 2000 (Illumina) using the TruSeq Rapid SBS Kit (\#FC-402-4001, Illumina). 


\section{Bioinformatic analysis of MBD-seq}

Sequenced reads were mapped to the reference human genome (GRCh37, hg19) using Bowtie (v0.12.7) [49]. Significantly enriched regions/peaks of methylation were determined using model-based analysis of ChIP-seq (MACS) algorithm [50], by comparing bound, enriched samples to input, non-enriched samples. Annotation was performed using ChIPpeakAnno R package (v.1.12.0) [51] and a customized version of Annovar program [52] with RefSeq genes to determine specific genomic features: promoter regions $(2.5 \mathrm{~kb}$ upstream and $500 \mathrm{bp}$ downstream from the nearest TSS), genic (further defined as 500 bp downstream from the nearest TSS), exons, introns, and intergenic (defined as greater than $100 \mathrm{~kb}$ up/downstream from the nearest TSS). UCSC CpG island definitions were used to define CpG islands. RWPE-1 DNase-seq data from the Encyclopedia of DNA Elements (ENCODE) project [GEO accession: GSM1008595] was correlated to MACS peak sets in both RWPE-1 and 22Rv1 cells. The genomic feature, RWPE-1 UCSC DNAseI peaks, was defined as within $5 \mathrm{~kb}$ of a DNase peak. The following options for ChiPpeakAnno were used: PeakLocForDistance="middle," FeatureLocForDistance $=$ "middle" for DNase I HS and CpG data, and FeatureLocForDistance="TSS" for RefSeq gene annotations. MACS data was further analyzed using DiffBind R package (v.1.12.0) [53] to determine three consensus peak sets by requiring that peaks from one, two, or all three replicates be present at the same location. The third consensus peak set was subsequently defined as differentially methylated regions (DMRs) between RWPE-1 and 22Rv1 cells (fold change $>1, \mathrm{FDR}<0.1$ ).

\section{Bioinformatic analysis of hMeSeal-seq}

Sequenced reads were mapped to the reference human genome (GRCh37, hg19) using Bowtie (v0.12.7) [49]. Repitools package [54] was used as an enrichment diagnostic screen of sequenced samples, enriched and input. Significantly enriched regions/peaks of hydroxymethylation were determined using model-based analysis of ChIP-seq (MACS) algorithm [50], by comparing bound, enriched samples to input, non-enriched samples. Annotation was performed using ChIPpeakAnno $\mathrm{R}$ package (v.1.12.0) [51] and a customized version of Annovar program [52] with RefSeq genes to determine specific genomic features: promoter, genic, exons, introns, and intergenic (previously described for MBD-seq). UCSC CpG island definitions were used to define CpG islands. RWPE-1 DNase-seq data from the ENCODE project [GEO accession: GSM1008595] was correlated to MACS peak sets in both RWPE-1 and 22Rv1 cells. The genomic feature, RWPE-1 UCSC DNAseI peaks, was defined as within $5 \mathrm{~kb}$ of a DNase peak. MACS data was further analyzed using DiffBind R package (v.1.12.0) [53] to determine two consensus peak sets by requiring one or both MACS peaks to be present for a consensus peak to be called. The first consensus peak set was subsequently defined as differentially hydroxymethylated regions (DHMRs) between RWPE-1 and 22Rv1 cells (fold change $>1$ ).

\section{Bioinformatic analysis of hMeDIP-seq}

Sequencing image analyses and base calling were performed using Off-Line Basecaller software (OLB V1.8). After passing Solexa CHASTITY quality filter, sequenced reads were mapped to the reference human genome (GRCh37, hg19) using Bowtie (V2.1.0) [49]. Significantly enriched regions/peaks of hydroxymethylation were determined using model-based analysis of ChIPseq (MACS, V2) algorithm [50], by comparing immunoprecipitated, enriched samples to input, non-enriched samples $\left(p\right.$ value $\left.<10^{-4}\right)$. Annotation was performed using the UCSC RefSeq database, where peaks were mapped to the nearest gene and specific genomic features were determined: promoter $(2500 \mathrm{~kb}$ upstream and 500 bp downstream from the TSS); gene body (500 bp downstream of the TSS to the TTS), including introns and exons; and intergenic (remaining regions not defined as "promoter" or "gene body" located more than $100 \mathrm{~kb}$ from the nearest TSS). UCSC CpG island definitions were used to define CPG islands. RWPE-1 DNaseseq data from the ENCODE project [GEO accession: GSM1008595] was correlated to MACS peak sets in both RWPE-1 and 22Rv1 cells. The genomic feature, RWPE-1 UCSC DNAseI peaks, was defined as within $5 \mathrm{~kb}$ of a DNase peak.

\section{Statistical analysis: correlation between genome-wide DNA methylation and hydroxymethylation marks} Specific regions/peaks from genome-wide MBD-Seq and hMeSeal-seq data where DNA hydroxymethylation overlapped methylation, hydroxymethylation overlapped hydroxymethylation, and methylation overlapped hydroxymethylation for RWPE-1 and 22Rv1, respectively, were identified and stratified by genomic feature as described previously. Significant proportional difference for modified regions from the expected proportion of overall RWPE-1 and $22 R v 1$ marks, such that the frequency of occurrence of modified regions could be explained by neither random change in RWPE-1 marks or by random distribution of 22Rv1 marks, was assessed using chi-square test.

\section{Statistical analysis: correlation between genome-wide DNA methylation or hydroxymethylation and gene expression}

Microarray RNA expression data were downloaded from GEO DataSets for RWPE-1 [GEO accession: GSM375783] and 22Rv1 [GEO accession: GSE36135]. Affymetrix probe identifications were linked to gene 
names using the $\mathrm{R}$ package (hgu133plus2.db, v.3.0.0), and a single expression value per gene was selected (a calculated average between replicates). The resulting dataset was sorted by expression and divided into three equal subsets/tiers of genes with low, medium, and high expression. Consensus peaks from MBD-seq (three sets) and hMeSeal-seq (two sets) analyses were intersected with each of the three tiers of genes, from microarray datasets. Peaks were obtained when found to overlap or flank genes with low (zero), moderate, or high expression. Each of the resulting datasets (15 sets) was further stratified into subsets of peaks differing in their location according to genomic features (previously defined for MBD-seq). In addition to the count of peaks for each of the peak sets defined above, the corresponding count of associated RefSeq coding genes was also recorded. The strength of association between peak or gene count and expression level was assessed using chi-square test, Pearson's correlation coefficient, and Fisher's exact two-tailed test, using $\mathrm{R}$ package (v.3.1.0). Fisher's exact test was performed comparing tier 1 (low expression) to tier 3 (high expression) gene/peak sets.

\section{Pathway analysis of MBD-seq and hMeSeal-seq datasets correlated with gene expression}

Genomic region lists were generated to represent significant differentially methylated or hydroxymethylated regions (DMRs or DHMRs), overlapping specific genomic features, identified by comparing RWPE-1 and 22Rv1 cells. DMR or DHMR lists were further stratified according to correlation with microarray RNA expression datasets (as described above). Pathway enrichment analysis was performed on the genomic region lists using the Genomic Regions Enrichment of Annotations Tool (GREAT) [55]. The background file submitted contained a complete list of total methylated or hydroxymethylated regions in both RWPE-1 and 22Rv1 datasets (no significance threshold applied). GREAT results were represented as an enrichment map $(p<0.05, \mathrm{FDR}<0.1$, Jaccard's similarity coefficient $<0.25$ ) [56] generated in the visualization software, Cytoscape (v3.1.0) [57]. Groups of related pathways (nodes) were identified using the MCODE cluster app and were then manually summarized (circled and labeled in figures) [58]. Additionally, GREAT results were organized into bar graphs according to the recommended statistic of $-\log _{10}$ (raw hypergeometric $p$ value) for ranking. Additionally, NGS (raw peaks) and microarray (raw gene expression) data were visualized using Circos software [59].

\section{Locus-specific DNA hydroxymethylation detection using hMeSeal-qPCR}

Following hMeSeal technique performed on a separate biological replicate from hMeSeal-Seq (previously described),
DNA hydroxymethylation levels were analyzed by RTqPCR using the 7500 Real-Time PCR Instrument (Applied Biosystems). Hydroxymethylation levels were calculated using $2^{-\Delta \Delta C t}$ method, relative to $2.7 \%$ of input genomic DNA, and normalized to $0.027 \%$ of input genomic DNA. A negative control reaction was performed according to the Hydroxymethyl Collector protocol (Active Motif), where UDP-azide-glucose was excluded from the glucosylation reaction. In addition, $2.5 \mu \mathrm{g}$ of sheared genomic DNA was subjected to each glucosylation reaction, for both sample and negative control reactions. The PCR assays comprised of $5 \mu \mathrm{l}$ of $\mathrm{PerfeCTa}^{\circ} \mathrm{SYBR}^{\circ}$ Green FastMix ${ }^{\circ}$, Low ROX (Quanta Biosciences), $2 \mu \mathrm{l}$ of bound DNA elution (hydroxymethylated or negative control), and $5 \mu \mathrm{M}$ of each primer, with a total volume of $10 \mu \mathrm{l}$. The 2.7 and $0.027 \%$ of input PCR assays were completed using the identical conditions, and input DNA stocks were prepared at 33.3 and $0.33 \mathrm{ng} / \mu \mathrm{l}$, respectively. All PCR assays included a non-template control, using UltraPure Distilled Water (Invitrogen) as the template. The PCR conditions were as follows: $30 \mathrm{~s}$ at $95{ }^{\circ} \mathrm{C}, 40$ cycles of denaturation for $5 \mathrm{~s}$ at $95^{\circ} \mathrm{C}$, and annealing for $30 \mathrm{~s}$ at $60^{\circ} \mathrm{C}$. Primers were designed using the specifications recommended by Hydroxymethyl Collector protocol (Active Motif) and targeted differentially hydroxymethylated regions (DHMRs) identified by both hMeSeal-seq and hMeDIP-seq. The sequences of primers amplifying: $R A S$ GEF1a are 5'-GCA TGT TCC TTG AAC TGT GA-3' (forward), 5'-TCA CAC CCT TCC CAA CAC TA-3'(reverse); HINT1 are 5' - CAT ATC CAA ATT GCC AGG AT-3' (forward), 5'- GCT GAC TTT GCT TTC AGA CC-3' (reverse); CUL2 are 5' -GGG GTG CAA TAT CTC ACT GT-3' (forward), 5'-GCT TGG AGA AGA CAC ACA AA-3' (reverse); and HOXD8 are $5^{\prime}$-AAC TTG CGG TCG TCT GCC CT-3' (forward), 5'-ACA GAA ACG TTC TGA GGC GGG AAA-3' (reverse). PCR reactions were performed across three technical replicates.

\section{Accession numbers}

The data sets supporting the results of this article are available in the Gene Expression Omnibus (GEO) repository, under accession number GSE74464.

\section{Additional file}

Additional file 1: Co-occurrence of methylation and

hydroxymethylation in pathway regulation. This file lists all pathways found to be co-occurrent between $5 \mathrm{mC}$ - and $5 \mathrm{hmC}$-enriched regions within each cell line, providing the genomic features and gene lists associated with enrichment for each mark.

Competing interests

The authors declare that they have no competing interests. 


\section{Authors' contributions}

SNK carried out the pathway analysis, performed hMeSeal-qPCR and data interpretation, and drafted the manuscript. LH performed $5 \mathrm{mC}$ and $5 \mathrm{hmC}$ enrichment, submitted sequencing samples, and helped to draft the manuscript. SNK and LH are considered as equal co-first authors in this study. $\mathrm{RH}$ and GB helped with pathway analysis and interpretation, and developed the MCODE and Enrichment Map apps used in the study. AZ, TvdK, and NH participated in design, coordination, and critical review of the manuscript. BB conceived of the study, participated in its design and coordination, and helped to draft the manuscript. All authors read and approved the final manuscript.

\section{Acknowledgements}

We thank Julia Garcia for her work performing dot blots for $5 \mathrm{hmC}$ detection in RWPE-1 and 22Rv1 cells.

The authors would like to acknowledge the support from the Bill and Kathleen Troost Innovation Grant of the Canadian Cancer Society (Grant \#701456), to BB, TvdK, and NF. LTH is funded by the University of Toronto (UofT) Fellowship, Lorne F. Lambier, Q.C. Scholarship (UofT), OSOTF Studentship at Mount Sinai Hospital (MSH), Paul Starita Graduate Student Fellowship (UofT), Research Training Centre (RTC) Travel Award at MSH, and the Laboratory Medicine and Pathobiology (LMP) Travel Award at U of T. SNK is funded by the Ontario Graduate Scholarship (OGS) - Faculty of Medicine at University of Toronto (UofT), Scace Graduate Fellowship in Prostate Cancer Research (UofT), George Sidney Brett Memorial Fund (UofT), Research Training Centre (RTC) Travel Award at MSH, and the Frank Fletcher Memorial Fund (UofT)

\section{Author details}

'Department of Laboratory Medicine and Pathobiology, University of Toronto, Toronto, ON, Canada. ${ }^{2}$ Mount Sinai Hospital, Lunenfeld-Tanenbaum Research Institute, Toronto, ON, Canada. ${ }^{3}$ The Donnelly Centre for Cellular and Biomolecular Research, University of Toronto, Toronto, ON, Canada. ${ }^{4}$ Department of Pathology, University Health Network, University of Toronto, Toronto, ON, Canada. ${ }^{5}$ Department of Surgery and Surgical Oncology, Division of Urology, University Health Network, University of Toronto, Toronto, ON, Canada. ${ }^{6}$ Division of Urology, University Health Network, Toronto, ON, Canada.

\section{Received: 11 November 2015 Accepted: 2 March 2016} Published online: 15 March 2016

\section{References}

1. Van Vlodrop IJ, Niessen HE, Derks S, Baldewijns MM, van Criekinge W, Herman JG, et al. Analysis of promoter CpG island hypermethylation in cancer: location, location, location! Clin Cancer Res. 2011. doi:10.1158/10780432. CCR-10-3394.

2. Esteller M. CpG island hypermethylation and tumor suppressor genes: a booming present, a brighter future. Oncogene. 2002;21(35):5427-40.

3. Sproul D, Kitchen RR, Nestor CE, Dixon JM, Sims AH, Harrison DJ, et al. Tissue of origin determines cancer-associated CpG island promoter hypermethylation patterns. Genome Biol. 2012. doi:10.1186/gb-2012-13-10-r84.

4. Esteller M, Herman JG. Cancer as an epigenetic disease: DNA methylation and chromatin alterations in human tumours. J Pathol. 2002;196(1):1-7.

5. Vilain A, Vogt N, Dutrillaux B, Malfoy B. DNA methylation and chromosome instability in breast cancer cell lines. FEBS Lett. 1999:460(2):231-4.

6. Sun W, Zang L, Shu Q, Li X. From development to diseases: the role of 5hmC in brain. Genomics. 2014;104(5):347-51.

7. Haffner MC, Chaux A, Meeker AK, Esopi DM, Gerber J, Pellakuru LG, et al. Global 5-hydroxymethylcytosine content is significantly reduced in tissue stem/progenitor cell compartments and in human cancers. Oncotarget. 2011;2(8):627-37.

8. Shukla A, Sehgal M, Singh TR. Hydroxymethylation and its potential implication in DNA repair system: a review and future perspectives. Gene. 2015;564(2):109-18.

9. Nestor CE, Ottaviano R, Reddington J, Sproul D, Reinhardt D, Dunican D, et al. Tissue type is a major modifier of the 5-hydroxymethylcytosine content of human genes. Genome Res. 2012;22(3):467-77.

10. Li W, Liu M. Distribution of 5-hydroxymethylcytosine in different human tissues. J Nucleic Acids. 2011. doi:10.4061/2011/870726
11. Khare T, Pai S, Koncevicius K, Pal M, Kriukiene E, Liutkeviciute Z, et al. 5-hmC in the brain is abundant in synaptic genes and shows differences at the exon-intron boundary. Nat Struct Mol Biol. 2012. doi: 10.1038/nsmb.2372

12. Sérandour AA, Avner S, Oger F, Bizot M, Percevault F, Lucchetti-Miganeh C, et al. Dynamic hydroxymethylation of deoxyribonucleic acid marks differentiation-associated enhancers. Nucleic Acids Res. 2012;40(17):8255-65.

13. Uribe-Lewis S, Stark R, Carroll T, Dunning MJ, Bachman M, Ito Y, et al. 5Hydroxymethylcytosine marks promoters in colon that resist DNA hypermethylation in cancer. Genome Biol. 2015. doi:10.1186/s13059-015-0605-5.

14. Stroud H, Feng S, Morey KS, Pradhan S, Jacobsen SE. 5-Hydroxymethylcytosine is associated with enhancers and gene bodies in human embryonic stem cells. Genome Biol. 2011. doi:10.1186/gb-2011-12-6-r54.

15. Vasanthakumar A, Godley LA. 5-hydroxymethylcytosine in cancer: significance in diagnosis and therapy. Cancer Genet. 2015. doi: 10.1016/j.cancergen.2015.02.009

16. Xu Y, Wu F, Tan L, Kong L, Xiong L, Deng J, et al. Genome-wide regulation of $5 \mathrm{hmC}, 5 \mathrm{mC}$, and gene expression by Tet1 hydroxylase in mouse embryonic stem cells. Mol Cell. 2011. doi:10.1016/j.molcel.2011.04.005.

17. Ficz G, Gribben JG. Loss of 5-hydroxymethylcytosine in cancer: cause or consequence? Genomics. 2014. doi: 10.1016/j.ygeno.2014.08.017

18. Mariani CJ, Madzo J, Moen EL, Yesilkanal A, Godley LA. Alterations of 5hydroxymethylcytosine in human cancers. Cancers (Basel). 2013. doi: 10. 3390/cancers5030786.

19. Turcan S, Rohle D, Goenka A, Walsh LA, Fang F, Yilmaz E, et al. IDH mutation is sufficient to establish the glioma hypermethylator phenotype. Nature. 2012. doi:10.1038/nature10866.

20. Xu W, Yang H, Liu Y, Yang Y, Wang P, Kim SH, et al. Oncometabolite 2hydroxyglutarate is a competitive inhibitor of a-ketoglutarate-dependent dioxygenases. Cancer Cell. 2011. doi:10.1016/j.ccr.2010.12.014

21. Jin SG, Jiang Y, Qiu R, Rauch TA, Wang Y, Schackert G, et al. 5hydroxymethylcytosine is strongly depleted in human cancers but its levels do not correlate with IDH1 mutations. Cancer Res. 2011. doi:10.1158/00085472.CAN-11-2023

22. Yang $\mathrm{H}$, Ye D, Guan $\mathrm{KL}$, Xiong $\mathrm{Y}$. IDH1 and IDH2 mutations in tumorigenesis: mechanistic insights and clinical perspectives. Clin Cancer Res. 2012. doi: 10.1158/1078-0432.CCR-12-1773.

23. Xiao M, Yang H, Xu W, Ma S, Lin H, Zhu H, Liu L, Liu Y, Yang C, Xu Y, Zhao S, Ye D, Xiong Y, Guan KL. Inhibition of a-KG-dependent histone and DNA demethylases by fumarate and succinate that are accumulated in mutations of FH and SDH tumor suppressors. Genes Dev. 2012. doi:10.1101/gad.191056.112

24. Haley H, Schultz N, Gopalan A, Carver BS, Chang MT, Xiao Y, et al. Copy number alteration burden predicts prostate cancer relapse. Proc Natl Acad Sci U S A. 2013;111(30):11139-44.

25. Luboldt HJ, Bex A, Swoboda A, Hüsing J, Rübben H. Early detection of prostate cancer in Germany: a study using digital rectal examination and 4. $0 \mathrm{ng} / \mathrm{ml}$ prostate-specific antigen as cutoff. Eur Urol. 2001;39:131-7.

26. Spiciarich D, Maund S, Peehl D, Bertozzi C. Identifying prostate cancer biomarkers by profiling glycoproteins in human prostate tissue. FASEB J. 2014;28:591.6.

27. Bangma CH, Roemeling S, Schröder F. Overdiagnosis and overtreatment of early detected prostate cancer. World J Urol. 2007;25(1):3-9.

28. Ruijter ET, van de Kaa CA, Schalken JA, Debruyne FM, Ruiter DJ. Histological grade heterogeneity in multifocal prostate cancer. Biological and clinical implications. J Pathol. 1996;180(3):295-9.

29. Abate-Shen C, Shen MM. Molecular genetics of prostate cancer. Genes Dev. 2000;14(19):2410-34

30. Fraser M, Berlin A, Bristow RG, van der Kwast T. Genomic, pathological, and clinical heterogeneity as drivers of personalized medicine in prostate cancer. Urol Oncol. 2015;33(2):85-94.

31. Brocks D, Assenov Y, Minner S, Bogatyrova O, Simon R, Koop C, Oakes C, Zucknick M, Lipka DB, Weischenfeldt J, Feuerbach L, Cowper-Sal LR, Lupien M, Brors B, Korbel K, Schlomm T, Tanay A, Sauter G, Gerhäuser C, Plass C. ICGC Early Onset Prostate Cancer Project. Intratumor DNA methylation heterogeneity reflects clonal evolution in aggressive prostate cancer. Cell Rep. 2014:8(3):798-806.

32. Yegnasubramanian S, Haffner MC, Zhang Y, Gurel B, Cornish TC, Wu Z, Irizarry RA, Morgan J, Hicks J, DeWeese TL, Isaavs WB, Bova GS, De Marzo AM, Nelson WG. DNA hypomethylation arises later in prostate cancer progression than $\mathrm{CpG}$ island hypermethylation and contributes to metastatic tumor heterogeneity. Cancer Res. 2008:68(21):8954-67.

33. Goessl C, Krause H, Müller M, Heicappell R, Schrader M, Sachsinger J, Miller K. Fluorescent methylation-specific polymerase chain reaction for DNA-based detection of prostate cancer in bodily fluids. Cancer Res. 2000;60(21):5941-5. 
34. Jerónimo C, Henrique R, Hoque MO, Mambo E, Ribeira FR, Varzim G, Oliveira J, Teixeira MR, Lopes C, Sidransky D. A quantitative promoter methylation profile of prostate cancer. Clin Cancer Res. 2004;10(24):8472-8.

35. Nestor CE, Ottaviano R, Reinhardt D, Cruickshanks HA, Mjoseng HK, McPherson RC, et al. Rapid reprogramming of epigenetic and transcriptional profiles in mammalian culture systems. Genome Biol. 2015. doi:10.1186/ s13059-014-0576-y

36. Boyle AP, Davis S, Shulha HP, Meltzer P, Margulies EH, Weng Z, et al. Highresolution mapping and characterization of open chromatin across the genome. Cell. 2008. doi:10.1016/j.cell.2007.12.014.

37. Bhattacharyya S, Yu Y, Suzuki M, Campbell N, Mazdo J, Vasanthakumar A, et al. Genome-wide hydroxymethylation tested using the HELP-GT assay shows redistribution in cancer. Nucleic Acids Res. 2013. doi:10.1093/nar/ gkt601.

38. Lercher L, McDonough MA, El-Sagheer AH, Thalhammer A, Kriaucionis S Brown $T$, et al. Structural insights into how 5-hydroxymethylation influences transcription factor binding. Chem Commun (Camb). 2014. doi:10.1039/ c3cc48151d

39. Michalakis S, Perera A, Eisen D, Laube SK, Wagner M, Müller M, et al. The epigenetic mark $5 \mathrm{hmC}$ recruits a dynamic set of readers in the retina (meeting abstract). Invest Ophthalmol Vis Sci. 2014;55:4997.

40. Rampal R, Alkalin A, Madzo J, Vasanthakumar A, Pronier E, Patel J, et al. DNA hydroxymethylation profiling reveals that WT1 mutations result in loss of TET2 function in acute myeloid leukemia. Cell Rep. 2014. doi:10.1016/j. celrep.2014.11.004

41. Lian CG, Xu Y, Ceol C, Wu F, Larson A, Dresser K, et al. Loss of 5hydroxymethylcytosine is an epigenetic hallmark of melanoma. Cell. 2012. doi:10.1016/j.cell.2012.07.033

42. Takayama K, Misawa A, Suzuki T, Takagi K, Hayashizaki Y, Fujimura T, et al. TET2 repression by androgen hormone regulates global hydroxymethylation status and prostate cancer progression. Nat Commun. 2015. doi:10.1038/ ncomms9219

43. Rhee SY, Wood V, Dolinski K, Draghici S. Use and misuse of the gene ontology annotations. Nat Rev Genet. 2008. doi:10.1038/nrg2363

44. Ura K, Obama K, Satoh S, Sakai Y, Nakamura Y, Furukawa Y. Enhanced RASGEF1a expression is involved in the growth and migration of intrahepatic cholangiocarcinoma. Clin Cancer Res. 2006;12(22):6611-6.

45. Shang B, Gao A, Pan Y, Zhang G, Tu J, Zhou Y, et al. CT45A1 acts as a new proto-oncogene to trigger tumorigenesis and cancer metastasis. Cell Death Dis. 2014. doi: 10.1038/cddis.2014.244.

46. Zhang YJ, Li H, Wu HC, Shen J, Wang L, Yu MW, et al. Silencing of Hint1, a novel tumor suppressor gene, by promoter hypermethylation in hepatocellular carcinoma. Cancer Lett. 2009. doi: 10.1016/j.canlet.2008.10.042

47. Klarmann GJ, Decker A, Farrar WL. Epigenetic gene silencing in the Wnt pathway in breast cancer. Epigenetics. 2008;3(2):59-63.

48. Park SW, Chung NG, Hur SY, Kim HS, Yoo NJ, Lee SH. Mutational analysis of hypoxia-related genes HIF1alpha and CUL2 in common human cancers. APMIS. 2009. doi:10.1111/j.1600-0463.2009.02550.x.

49. Langmead B, Trapnell C, Pop M, Salzberg SL. Ultrafast and memory-efficient alignment of short DNA sequences to the human genome. Genome Biol. 2009. doi:10.1186/gb-2009-10-3-r25

50. Zhang Y, Liu T, Meyer CA, Eeckhoute J, Johnson DS, Bernstein BE, et al. Model-based analysis of ChIP-Seq (MACS). Genome Biol. 2008. doi:10.1186/ gb-2008-9-9-r137

51. Zhu LJ, Gazin C, Lawson ND, Pagès H, Lin SM, Lapointe DS, et al. ChIPpeakAnno: a Bioconductor package to annotate ChIP-seq and ChIPchip data. BMC Bioinformatics. 2010. doi:10.1186/1471-2105-11-237

52. Wang K, Li M, Hakonarson $H$. ANNOVAR: functional annotation of genetic variants from high-throughput sequencing data. Nucleic Acids Res. 2010. doi:10.1093/nar/gkq603

53. Stark R, Brown G. DiffBind: differential binding analysis of ChIP-Seq peak data. 2011. http://bioconductor.org/packages/release/bioc/vignettes/ DiffBind/inst/doc/DiffBind.pdf. Accessed 2 August 2015

54. Statham AL, Strbenac D, Coolen MW, Stirzaker C, Clark SJ, Robinson MD. Repitoold: an R package for the analysis of enrichment-based epigenomic data. Bioinformatics. 2010. doi:10.1093/bioinformatics/bta247.

55. McLean CY, Bristor D, Hiller M, Clarke SL, Schaar BT, Lowe CB, et al. GREAT improves functional interpretation of cis-regulatory regions. Nat Biotechnol. 2010. doi:10.1038/nbt.1630
56. Merico D, Isserlin R, Stueker O, Emili A, Bader GD. Enrichment map: a network-based method for gene-set enrichment visualization and interpretation. PLoS One. 2010. doi:10.1371/journal.pone.0013984.

57. Cline MS, Smoot M, Cerami E, Kuchinsky A, Landys N, Workman C, et al. Integration of biological networks and gene expression data using Cytoscape. Nat Protoc. 2007;2(10):2366-82.

58. Bader GD, Hogue CW. An automated method for finding molecular complexes in large protein interaction networks. BMC Bioinformatics. 2003. doi:10.1186/1471-2105-4-2

59. Krzywinski M, Schein J, Birol I, Connors J, Gascoyne R, Horsman D, et al. Circos: an information aesthetic for comparative genomics. Genome Res. 2009. doi:10.1101/gr.092759.109

\section{Submit your next manuscript to BioMed Central and we will help you at every step:}

- We accept pre-submission inquiries

- Our selector tool helps you to find the most relevant journal

- We provide round the clock customer support

- Convenient online submission

- Thorough peer review

- Inclusion in PubMed and all major indexing services

- Maximum visibility for your research

Submit your manuscript at www.biomedcentral.com/submit

) Biomed Central 